\title{
Mode of delivery and weight shape the intestinal microbiome composition progression in preterm infants: results of a prospective study
}

Fardou Heida ( $\nabla$ fardou@gmail.com )

https://orcid.org/0000-0002-4497-7513

\section{Elisabeth Kooi}

Unversity Medical Center Groningen, Neontology

Thi-Yen Nguyen

University Medical Center Groningen, Pediatric Surgery

Jan Hulscher

University Medicla Center Groningen, Pediatric Surgery

Anne van Zoonen

University Medical Center Groningen, Pediatric Surgery

Arend Bos

University medical Center Groningen, Neonatology

Hermie Harmsen

University Medical Center Groningen, Medical Microbiology

Marcus de Goffau

University of Cambridge Department of Veterinary Medicine

\section{Research}

Keywords: intestinal microbiome, prematurity, development, weight

Posted Date: February 24th, 2020

DOI: https://doi.org/10.21203/rs.2.24238/v1

License: (1) (1) This work is licensed under a Creative Commons Attribution 4.0 International License. Read Full License 


\section{Abstract}

Background: In preterm infants the intestinal microbiome differs markedly from term infants. However, it is unclear whether the microbiome follows infant specific maturation patterns or whether it is mainly characterized by varying states of dysbiosis. We investigated the development of the intestinal microbiome in extremely preterm infants over time by $16 \mathrm{~S}$ rRNA amplicon sequencing. We analysed the first meconium and faecal samples from the $2 \mathrm{nd}, 3 \mathrm{rd}$ and 4 th weeks, and (clinical) metadata to identify the main factors influencing the microbiota composition development.

Results: The study included 41 extremely preterm infants (gestational age 25-30 weeks; birth weight (BW) 430-990g). Birth via Caesarean section (CS) was associated with placental insufficiency during pregnancy and lower BW. In meconium and in weeks 2 and 3 an increased combined abundance of Escherichia and Bacteroides (maternal aerotolerant fecal bacteria) was associated with vaginal delivery ( $p=0.039, p=0.0002, p=0.034$, respectively) while Staphylococcus epidermidis (skin bacterium) was associated with CS ( $p=0.001, p=0.0003 p=0.048$, respectively). Secondly, a switch was observed from a microbiome dominated by $\mathrm{S}$. epidermidis (Bacilli) towards a microbiome dominated by Enterobacteriaceae (Gammaproteobacteria, mainly represented by Klebsiella and Escherichia ), in which the stage of progression appeared to be dependent upon the current weight of the infant, irrespective of the week of sampling or the mode of birth.

Conclusions: Our data shows that the mode of delivery does affect the meconium microbiome composition. It also suggests that the weight of the infant at the time of sampling is a better predictor for the stage of progression of the intestinal microbiome development/maturation than gestational/postnatal age. We hypothesize that impaired growth, for example due the effects of diminished placental function during pregnancy, is a key factor in the maturation of the intestinal microbiome in extreme premature infants.

\section{Introduction}

Extreme prematurity involves high mortality and morbidity [1]. Diseases such as necrotizing enterocolitis (NEC) are related to prematurity with aberrant gut microbiome colonization patterns [2-4]. For example, NEC has been associated with a particular group of clostridial species closely related to Clostridium perfringens in many studies, while staphylococci appear to be associated with a decreased risk for NEC development [5-13].

Previous studies on the intestinal microbiome of preterm infants, using 16S rRNA-based sequencing technologies, have revealed remarkable differences with the microbiome of term infants, including higher abundances of bacilli and Gammaproteobacteria [14-26]. The process of microbiota maturation, a pattern where microbiome maturation is mirrored by the maturation of the infant, is important in understanding differences between the microbiome of preterm- and term infants. In preterm infants, the 
prolonged absence of bacteria that usually colonize and protectterm infants (bifidobacteria) is a clear indicator that the intestinal maturation process is either severely disturbed or altered [5,6,21,26-29].

Besides host biology, such as low birth weight (BW) and immaturity of the multiple organs including the gastrointestinal tract as a result of low gestational age (GA), there are multiple other exogenous factors that could affect the intestinal microbiome development/maturation of preterm infants (i.e. mode of delivery, neonatal feeding regime, the neonatal intensive care unit (NICU) environment and peri- and postnatal antibiotic exposure) $[5,6,14,16,19,29]$.

While interest in this subject is growing, the focus of research is typically, with a few exceptions [15-19, 23], on the relation between the intestinal microbiome of the preterm infant and disease instead of on microbiome composition development itself. We aimed to determine whether the early microbiome development in extremely premature infants born between 25 and 30 weeks of gestation is dependant by infant maturity/maturation or whether it is mainly characterized by varying states of dysbiosis. Secondly, we aimed to analyse whether exogenous factors were intricately linked with microbiome maturation.

\section{Results}

\section{Patient characteristics}

In this study 41 preterm born infants were included. The GA ranged between 25 and 30 weeks (median 27.6 weeks IQR 26.0-28.1). Table 1 summarizes the baseline characteristics. Detailed patient characteristics per week are shown in Table 2. Important for understanding this cohort, the mode of delivery was strongly associated with the birth weight Z-score for GA and gender (Figure 1a). Infants delivered by CS had a higher GA on average than their vaginally born counterparts (median 28.1 IQR 26.7-28.2) vs 26.0 IQR 25.3-26.9 weeks, $p=0.002$ ), yet were of lower BW (median 778 IQR 641- 923 vs 835 IQR $800-980$ grams, $p=0.03$ ). Underlying causes of prematurity for all vaginally delivered infants $(13 / 13)$ were intra-uterine infection, (prolonged) premature rupture of membranes and/or cervix insufficiency while most infants delivered by C-section were born preterm because of placental insufficiency (22/27) (Table 1). Placental insufficiency was negatively associated with the Z-score for birthweight $(p=0.0005$, Figure $1 b)$.

\section{Intestinal microbiome development over time}

We analyzed 142 samples (3.5 samples per patient on average, range 2-4), including the first meconium. An overview of the abundances (\% of reads per sample) of the most important bacterial groups (species/genera) in these 4 timepoints is shown in Figure 2. Within meconium samples Staphylococcus epidermidis was most frequently the most abundant bacterial species ( $11 / 37$ samples, median $14.6 \%$ IQR $1.0 \%-39.8 \%$ ), followed by Klebsiella oxytoca (10/37 samples, median $11.4 \%$ IQR $1.0 \%-31.0 \%$ ). On the family level Enterobacteriaceae, mainly represented by Klebsiella oxytoca, Klebsiella pneumonia and Escherichia coli were the most abundant (20/37 samples, median 37.2\% IQR 3.5\%-58.0\%) in meconium. 
In week 2 a large increase in the abundance of staphylococci was observed in this cohort with $S$. epidermidis becoming the dominant species in the majority of samples (20/37 samples, median $55.9 \%$ IQR $13.8 \%-82.7 \%$ ). A decline however of $S$. epidermidis is observed in weeks 3 and 4 (median $5.2 \%$ IQR $0.2 \%-26.5 \%$ and median $2.3 \%$ IQR $0.2-11.0 \%$, respectively) with Enterobacteriaceae becoming dominant again in most samples (median 53.3\% IQR 1.5\%-74.6\% and median 63.2\% IQR 0.4-92.5\%, respectively). Bifidobacteria and lactobacilli were groups of minor importance within this cohort.

\section{Mode of delivery and intestinal microbiota development}

One of the most striking patterns in the data, alluded to in Figure 2, is that the mode of delivery has a significant influence on the microbiome composition. This was most evident within the first three weeks after birth (Figure 3). Abundance of $S$. epidermidis, a typical skin bacterium, was significantly associated with CS delivery in samples from the first 3 weeks of life $(p=0.001, p=0.0003 p=0.048$ and $p=0.22$ for weeks $1-4$, respectively). The combined prevalence the facultative anaerobe $E$. coli and members of the aero-tolerantanaerobic Bacteroides genus (B. fragilis, B. vulgatus, $B$. dorei, B. thetaiotaomicron, $B$. uniformis \& B. ovatus),, typical maternal fecal representatives, were significantly associated with vaginal delivery during these first 3 weeks $(p=0.039, p=0.0002, p=0.034$ and $p=0.95$ for weeks $1-4$, respectively).

\section{Mode of delivery and infant weight development}

In this cohort the mode of delivery was significantly associated with BW and GA (Figure 1). The association between the infant's current weight at sampling time and the mode of delivery remains largely unchanged during the first four weeks $(p=0.04, p=0.03, p=0.02$ and $p=0.04$ for weeks $1-4$, respectively), as no difference was present in weight gain in $\mathrm{g} /$ week of infants delivered vaginally and by CS $(p=0.9 ; p=0.8 ; p=0.6$ for weeks $2-4$, respectively). Z-scores from BW were strongly correlated with absolute weight in all 4 weeks (Spearman $\otimes$ correlation coefficients of $0.70,0.67,0.66$ and 0.52 , respectively).

\section{Infant weight and intestinal microbiome development}

While the mode of delivery was the most important determinant for the infants' initial microbiome (Figures 2 and 3), the increase of Enterobacteriaceae in weeks 3 and 4 appeared associated with absolute weight at sampling time (Figure 4). When comparing infants with an above-median weight (835g) with their lighter counterparts, little difference was observed in the microbiome composition of the meconium (week 1). In week 2, infants with an above median weight (860g) contained significantly less $S$. epidermidis $(\mathrm{p}=0.013)$. This particular association was however partially indirect, as infants delivered by CS had a lower median BW and were more frequently colonized during delivery by $S$. epidermidis (Figure 3a). The weight of infants increased from a median of $860 \mathrm{~g}$ in week 2 to $969 \mathrm{~g}$ in week 3 to $1095 \mathrm{~g}$ in 
week 4, respectively. In week 3 Enterobacteriaceae became more dominant again at the expense of $S$. epidermidis, as the influence of the mode of delivery declined. In week 4 the correlation coefficient between abundance of Enterobacteriaceae and current weight was significant $(\mathbb{Q}=0.4, p=0.04)$. More specifically, at a body weight of $>1100 \mathrm{~g}$ nearly all samples were dominated by Enterobacteriaceae. In general, the absolute weight of infants, irrespective of the week of sample collection, appeared to be (independently) associated with the shift from a S. epidermidis dominated gut towards one dominated by Enterobacteriaceae (Figure 5). When combining all samples from all 4 timepoints, S. epidermidis was negatively associated with absolute weight measured at sampling time $(\mathbb{V}=0.39, p=0.000001)$ while the abundance of Enterobacteriaceae correlated positively with absolute weight measured at sampling time $(\nabla=0.25, p=0.003)$.

\section{Intestinal microbiota development and health}

While staphylococci were associated with low absolute weight in this cohort, they did not appear detrimental to health as 1) the amount of weight gain during any single week did not correlate with the gut microbiome composition (or any of the individual species) at the start of that week and 2) their previously reported negative association with necrotizing enterocolitis development was similarly found within this cohort in meconium samples $(p=0.034)$. Overall infant mortality in this cohort, in part caused by necrotizing enterocolitis ( $n=8$ in total; 3 caused by NEC), was not significantly associated with the gut microbiome composition or with BW $(p=0.36)$ but it was negatively associated with GA at birth $(p=$ $0.005)$ and positively but not significantly $(p=0.08)$ with prolonged premature rupture of membranes (PPROM).

\section{Exogenous factors and microbiota development}

Exogenous factors such as antibiotics use and/or feeding regime were found to be of ancillary importance in comparison with patterns associated with the mode of delivery or with absolute body weight. Associations of bacterial groups with antibiotics use were either found to be non-significant or disappeared when adjusting the analyses for mode of delivery. The current number of subjects was insufficient to unravel significant associations between microbiome development with antibiotics and feeding regimes.

\section{Discussion}

This study, which prospectively investigated the development of the microbiome of extremely preterm infants during the first four weeks of life, has three main findings. First, confirming current data [30], differences caused by mode of delivery have a strong but transient influence on intestinal microbiome composition. Importantly, this effect can already be observed in the meconium, in contrast to findings of others [31] who perhaps do not properly correct for reagent contamination in low biomass samples [32]. Second, our data suggest that there are weight thresholds, which determine the stage of the progression 
in maturation and development of the colonization process. There is a transition from a microbiome with a high abundance of $\{1\}$ staphylococci (bacilli) in extremely preterm infants (<1000 g) towards one dominated by $\{2\}$ Enterobacteriaceae as they gain weight. Third, this study reveals that current absolute weight is a better marker for the maturation of the infants' intestinal microbiome than postconceptional age as it less confounded by various infant-specific factors.

Recently, La Rosa et al [26] and Korpela et al [33] both described the hypothesis that the gut microbiome of the preterm infant appears to follow a patterned progression linked with postconceptional age as a key marker for host biology / maturity. La Rosa, et al. [26] describes a progression from $\{1\}$ bacilli to $\{2\} \mathrm{Y}^{-}$ Proteobacteria (Enterobacteriaceae) to $\{3\}$ clostridia (and Negativicutes). In this hypothesis, the place where preterm infants step into this progression is mainly dependent on their GA at birth [26]. A longer follow-up study would have seen a progression into $\{4\}$ Bifidobacterium (and/or Bacteroides).. Our study, which focused on the first 4 weeks of life of extremely preterm infants, confirmed the first part of this transition, namely the progression of a staphylococci (bacilli) dominance tot an Enterobacteriaceae dominated microbiome in extremely preterm infants. We however found that gestational/postnatal age was an inaccurate descriptor of maturity/host biology with regard to the development of the gut microbiome. A reason for this inaccuracy is highlighted by our comparison of infants delivered vaginally or by CS. In our cohort CS delivered infants had on average a higher GA at birth but a lower BW (Z-score) than their vaginally delivered counterparts (Figure 1). In this dataset the type of pregnancy complication (placental insufficiency vs intra-uterine infection/spontaneous preterm birth) was not only significantly associated with the mode of delivery but also with Z-scores for BW, representing fetal growth restriction.

In the cohort observed by Ho et al. [16] they similarly found that bacilli and Enterobacteriaceae formed the dominant groups but they ascribed their findings to a dichotomous development of the gut microbiome. The dichotomous development of the gut includes one cluster (I) of samples starting off with a high abundance of staphylococci which in time gave way to an increased abundance of Enterobacteriaceae and the other cluster (II) starting off with a high abundance of Enterobacteriaceae that declined slowly as clostridia became more prominent [16]. The developments in these two clusters fit perfectly into the aforementioned patterned progression if weight is used as a marker of intestinal microbiome maturity instead of gestational/postnatal age. Instead of a threshold for GA, which La Rosa [26] suspected, there might be a weight-determined threshold, which influences the gut microbiome maturation. In the cohort of Ho et al., infants from cluster I and II had a similar GA at birth on average (28.0 vs. 27.9) but infants assigned to cluster I had a BW of $1053 \mathrm{~g}$ while those assigned to cluster II had a BW of $1176 \mathrm{~g}$ [16]. In our study we found that the switch (threshold) between staphylococci and Enterobacteriaceae was particularly evident around $1100 \mathrm{~g}$, consistent with the difference between these 2 clusters.

The main driver for this difference is the cause of prematurity; placental insufficiency is typically accompanied by C-section and is logically negatively associated with lower BW Z-scores due to fetal growth restriction of the infant. Furthermore, some infants thrive and concomitantly gain weight after birth while others do not, although both increase equally in gestational/postnatal age. We observed that absolute weight, as a logical key marker for host biology, does not suffer from these confounders and 
could be used to clearly visualize the patterned progression of $\{1\}$ staphylococci to $\{2\}$ Enterobacteriaceae (Figure 5) in this dataset. The underlying mechanism why weight influences the gut microbiome maturation is not understood. We hypothesize that fetal growth restriction / low (birth) weight influences immune response modulation and altered intestinal development (i.e. influencing maturation of paneth cells, mucus production) that could cause weight dependent microbiome maturation differences [34].

Exogenous factors other than the mode of delivery such as antibiotics, neonatal feeding regime were also analyzed with regard to their association with the intestinal microbiome but no significant correlations were found in this cohort. While such external factors have been found to be associated with certain aspects of the gut microbiome development, they do not appear to represent the main drivers of gut maturation in preterm infants. More samples would be required to study these factors. Maternal data, such as the results of vaginal swabs and/or the use of maternal antibiotics, were not available for this study but could be of possible relevance for initial colonization.

This study highlights that the intestinal gut microbiome development in the extremely preterm infants is mainly driven by weight but is initially strongly affected by the mode of delivery. It also stresses the importance of pregnancy complications with diminished placental function as it directly affects the actual physical maturity level of the infant in which in turn directly affects the stage of progression [26; 33] from which the gut microbiome development starts. Underlying mechanism behind weight thresholds is not yet understood. There is a need for more knowledge on the affect of (birth) weight on immunological responses and organ maturation.

Before interventions are implemented, such as targeted antibiotic therapies or the use of pre- and probiotics, it is critical to understand which organisms are to be considered normal (part of the maturation process), in regards to the level of gut development of the infant at a certain weight, and which ones are indicative of potential dysbiosis/disease. For example, K. oxytoca or K. pneumonia, which belong to the family Enterobacteriaceae, have been frequently associated with, amongst other things, sepsis in newborns, but they appear to be a 'normal' part of the patterned progression of the bacteria composition as the infant gut matures $[16,26]$. Trials with i.a. strain resolved metagenomic analyses of a larger number of samples might show that only particular Klebsiella strains are to be associated with disease directly or indirectly. Colonization with Klebsiella appears to be normal whereas sepsis with Klebsiella might merely be a symptom of other things going wrong in the preterm infants' gut $[35,36]$. Our results underline the importance of larger multi-center observational studies to reveal the exact intestinal microbiome maturation of the extremely preterm infant and its underlying driving factors, such as immunomodulation.

\section{Conclusion}

During the first four weeks of life the gut microbiome of extremely preterm infants (birth weight $<1000$ ) undergoes a transition from a gut microbiome with a high abundance of staphylococci towards one dominated by Enterobacteriaceae. This study improves the patterned progression hypothesis [26] by 
stressing the importance of the infants' absolute weight at sampling time above gestational/postnatal age as an accurate marker of host biology and maturation. These findings were independent of pregnancy complications with diminished placental function. We hypothesize that weight thresholds determine the stage of the progression in maturation and development of the colonization process. Exogenous factors were in this cohort, apart from the strong yet transient effect of the mode of delivery, of minor importance.

\section{Methods}

This study is part of a prospective observational trial (CALIFORNIA trial; registered as NTR4153 in the Dutch trial registry, and approved by the Medical Ethics Committee of the University Medical Center Groningen), which studied infants for developing NEC [5,6,37-40]. The CALIFORNIA trial included 100 infants admitted to the Neonatal Intensive Care Unit of the University Medical Center Groningen between October 2012 and December 2014, after informed consent of their parents was given. Infants born at a GA of $\leq 30$ weeks and born with a BW of $\leq 1000$ grams were applicable for the study. Patients with other abdominal diseases (abdominal wall defects or congenital intestinal atresia) were excluded from this trial.

\section{Patients}

We selected infants from the CALIFORNIA trial who were born at a GA of $\leq 30$ weeks and / or who had a BW of $\leq 1000$ grams, from whom more than two samples during the first 4 weeks were available. Patient characteristics and demographic variables were derived from the CALIFORNIA database.

\section{Demographic and clinical variables}

Data from each sample day were used. Variables consisted of mode of delivery, BW, GA at birth, z-score BW (which represents the standard deviation in SD units from the Dutch reference growth curves) [41], bodyweight on the sample day, the administration of mothers' milk and / or of formula milk in milliliters/kg on the sample day, the antibiotic use on the sample day and if antibiotics were administered for more than 48 hours after birth. Complications during pregnancy were classified as placental insufficiency (pre-eclampsia/HELLP, fetal growth restriction and fetal distress) and intra-uterine infection/spontaneous preterm birth (chorioamnionitis, PPROM, cervical insufficiency and premature contractions).

\section{Faecal samples}

We intended to analyze one fecal sample per week, starting with the first meconium, and afterwards the first fecal sample of every week during the first four weeks after birth. Faecal samples were stored at- 
$80^{\circ} \mathrm{C}$ prior to the start of this study. The first sample of each week was used for analyses of the current sub-study.

\section{DNA extraction and sequence library preparation}

Faecal DNA was extracted from a $0.25 \mathrm{~g}$ faecal sample by double bead beating in combination with the QIAamp DNA Mini kit (Qiagen; Hilden, Germany), as described by a study that used the same technique [42]. Polymerase chain reaction (PCR) amplification targeted the V3 and V4 region of the 16S rRNA gene by using modified $341 \mathrm{~F}$ and $806 \mathrm{R}$ primers [43-44]. The $806 \mathrm{R}$ primers contained a 6-nucleotide barcode. An detailed description of the PCR reaction, DNA cleanup, and MiSeq library preparation is found in the Appendix file 1.

\section{Analyses of sequence reads}

The software used to analyze the data received from Illumina paired-end sequencing included PANDAseq [45] and ARB [46]. Readouts with a quality score lower than PANDAseq as standard practice to increase the quality of the sequence readouts discarded 0.9. ARB was used to identify sequences to the species level. As particularly meconium samples, but also many later samples have low bacterial biomasses, reagent contamination needs to be accounted for. Reagent contamination recognition analyses were subsequently performed as described by de Goffau et al [42], using the Spearman's rank correlation coefficients method. The consistency of the ratio of reagent-derived species within samples allows for their rapid identification. As a result, all reads identified as Undibacterium oligocarboniphilum, Variovorax paradoxus, Sphingomonas oligophenolica, Ralstonia pickettii, Curvibacter lanceolatus, Ralstonia insidiosa, Erythrobacter aquimaris, Afipia genosp., Ochrobactrum pseudintermedium, Sphingomonas mucosissima, Brevundimonas vesicularis, Arthrobacter russicus and Pelomonas saccharophila were removed before further analysis.

\section{Statistics}

Statistical analyses were conducted with IBM SPSS Statistics 21.0. Combinations of principal component analysis (PCA), regression and paired analyses were performed to examine the relationship between the microbiota and the following factors: mode of delivery, the birth weight, gestational age at birth, z-score birth weight, bodyweight on the sample day, the administration of mothers' milk and / or of formula milk in milliliters $/ \mathrm{kg}$ on the sample day, the antibiotic use on the sample day and antibiotic use for more than $48 \mathrm{~h}$ after birth. Two sided P-values less than 0.05 were considered statistically significant. Unless otherwise indicated, the Mann-Whitney-U test or Chi-square test were used to test differences between groups. Testing of the correlation between parameters was done with the Spearman's correlation test, while one-way-ANOVA was used to assess individual parameters development in time. 


\section{References}

1. Dahl C, Stigum H, Valeur J, Iszatt N, Lenters V, Peddada S, et al. Preterm infants have distinct microbes not explained by mode of delivery, breastfeeding duration or antibiotic exposure. Intl $\mathrm{J}$ Epidemiol 2018: doi.org/10.1093/ije/dyy064.

2. Claud EC, Keegan KP, Brulc JM, Lu L, Bartels D, Glass E, et al. Bacterial community structure and functional contributions to emergence of health or necrotizing enterocolitis in preterm infants. Microbiome 2013; 1.

3. Rich BS, Dolgin SE. Necrotizing entercolitis. Pediatr Rev 2017; 38(12).

4. Putignani L, Del Chierico F, Petrucca A, Vernocchi P, Dallapiccola B. The human gut microbiota: a dynamic interplay with host from birth to senescence settled during childhood: Pediatr Res 2014; 76.

5. Heida FH, van Zoonen AGJF, Hulscher JBF, te Kiefte BJC, Wessels R, Kooi EMW, et al. A Necrotizing enterocolitis-associated gut microbiota is present in the meconium: results of a prospective study. Clin Infect Dis 2016; 62(7).

6. Heida FH, van Zoonen AGJF, Hulscher JBF, te Kiefte BJC, Wessels R, Kooi EMW,et al. Reply to Cassir, et al. Clin Infec Dis 2016; 62(7).

7. Cassir N, Benamar S, Khalil JB, Croce O, Saint-Faust M, Jacquot A, et al. Clostridium butyricum strains and dysbiosis linked to necrotizing enterocolitis preterm neonates. Clin Infec Dis 2015; 61(7).

8. Alfa MJ, Robson D, Davi M, Bernard K, van Caeseele P, Harding GKM. An outbreak of necrotizing enterocolitis associated with a novel clostridium species in a neonatal intensive care unit. Clin Infect Dis 2022; 35: S101-5.

9. Sim K, Shaw AG, Randfell P, Cox MJ, McClure ZE, Li MS, et al. Dysbiosis anticipating necrotizing enterocolitis in very premature infants. Clin Infect Dis 2005; 60(3).

10. Kliegman RM, Fanaroff AA, Izant R, Speck WT. Clostridia as pathogens in neonatal necrotizing enterocolitis. J Pediatr 1979; 95.

11. Dittmar E, Beyer P, Fischer D, Schafer V, Schoepe H, Bauer K, et al. Necrotizing enterocolitis of the neonate with Clostridium perfringens: diagnosis, clinical course, and role of alpha toxin. Eur $\mathrm{J}$ Pediatr $2008 ; 167$.

12. Zhou Y, Shan G, Sodergren E, Weinstock G, Walker WA, Gregory KE. Longitudinal analysis of the premature infant intestinal microbiome prior to necrotizing enterocolitis: a case-control study. PLoS One 2015; 10: e0118632.

13. De La Cochetiere MF, Piloquet H, des Robert C, Darmaun D, Galmiche P, Roze JC. Early intestinal bacterial colonization and necrotizing enterocolitis in premature infants: the putative role of Clostridium. Pediatr Res 2004; 56.

14. Grier A, Qiu X, Bandyopadyay S, Holden-Wiltse J, Kessler HA, Gill AL. Impact of prematurity and nutrition on the developing gut microbiome and preterm infant growth. Microbiome 2017; 15: 158. Doi: 10.1186/540168-017-0377-0. 
15. Gómez M, Moles L, Espinose-Martos I, Bustos G, de Vos WM, Fernandez L, et al. Bacteriological and immunological profiling of meconium and fecal samples from preterm infants: a two-year follow-up study. Nutrients $2017 ; 9(12)$.

16. Ho TTB, Groer MW, Kane B, Yee AL, Torres BA, Gilbert JA, Maheshwari A. Dichotomous development of the gut microbiome in preterm infants. Microbiome 2018 ; 6(157) : doi: 10.1186/s40168-0180547-8

17. Alcon-Giner C, Caim S, Mitra S, Ketsemety J, Wegmann U, Wain J, et al. Optimisation of 16s rRNA gut microbiota profiling of extremely low birth weight infants. BMC Genomics 2017; 18.

18. Chernikova DA, Madan JC, Housman ML, Zain-Ul-Abideen M, Lundgren SN, Morrison HG, et al. The premature infant gut microbiome during the first 6 weeks of life differs based on gestational maturity at birth. Pediatr Res 2018.

19. Ardisonne AN, de la Cruz DM, Davis-Richardson AG, Rechcigl KT, Li N, Drew JC, et al. Meconium microbiome analysis identifies bacteria correlated with premature birth. Plos One 2014; 9(3): e90784.

20. Morowitz MJ, Poroyko V, Caplan M, Alverdy J, liu DC. Redefining the role of intestinal microbes in the parthogenesis of necrotizing enterocolitis. Pediatrics 2010; 125.

21. Underwood MA, Sohn K. The microbiota of the extremely preterm infant. Clin Perinatol 2017; 44(2).

22. Melville JM, Moss TJM. The immune consequences of preterm birth. Front neurosci 2013. 7(79).

23. Moles L, Gomes M, Heilig H, Bustos G, Fuentes S, de Vos W, et al. Bacterial diversity in meconium of preterm neonates and evolution of their fecal microbiota during the first month of life. PLoS one 2013; 8(6); e66986.

24. Butel MJ, Suau A, Campeotto F, Magne F, Aires J, Ferraris L, et al. Conditions of bifidobacterial colonization in preterm infants: a prospective analysis. J Pediatr Gastroenterol Nutr 2007; 44.

25. Drell T, Lutsar I, Stsepetova J, Parm U, Metsvaht T, Ilmoja ML, et al. The development of gut microbiota in critically ill extremely low birth weight infants assessed with 16S rRNA gene based sequencing. Gut Microb 2014; 5(3).

26. La Rosa PS, Warner BB, Zhou Y, Weinstock GM, Sodergren E, Hall-Moore CM, et al. Patterned progression of bacterial populations in the premature infant gut. Proc Natl Acad Sci USA 2014; 111(34).

27. Jandhyala SM, Talukdar R, Subramanyan C, Vuyyuru H, Sasikala M, Nagheshwar reddy D. Role of the normal gut. World J Gastrioneterol 2015; 21(29).

28. Stiemsa LT, Mickels KB. The role of the microbiome in the developmental origins of health and disease. Pediatrics 2018; 141(4): e20172437.

29. Martin R, Makino H, Cetinyurek Yavuz A, Ben-Amor K, Roel ofs M, Ishikawa E, et al. Early-life events, including mode of delivery and type of feeding, sibilings and gender, shape the developing gut microbiota. PLoS One 2016; 11(6): e0158498.

30. Shap Y, Forster SC, Tsaliki E, Vervier K, Strang A, Simpson N, et al. Stunted microbiota and opportunistic pathogen colonization in caesarean-section birth. Nature 2019; 574. 
31. Liu CJ, Liang X, Niu ZY, Jin Q, Zeng XQ, Wang WX, et al. Is the delivery mode a critical factor for the microbial communities in the meconium? Ebiomed 2019: 49.

32. de Goffau MC, Lager S, Salter SJ, Wagner J, Kronbichler A, Charnock-Jones DS, et al. Recognizing the reagent microbiome. Nature Microbiology 2018: 3.

33. Korpela K, Blakstad EW, Moltu SJ, Strommen K, Nakstad B, Ronnestad AE, et al. Intestinal microbiota development and gestational age in preterm neonates. Sci Rep 2018; 8(1).

34. Malhotra A, Allison BJ, Castillo-Melendez M, Jenkin G, Polglase GR, Miller SL. Neonatal morbidities of fetal growth restriction: pathophysiology and impact. Front Endocrinol 2019: 10; 55: doi: 10.3389/fendo.2019.00055.

35. Reiss I, Borkhardt A, Füssle R, Sziegoleit A, Gortner L. Disinfectant contaminated with Klebsiella oxytoca as a source of sepsis in babies. Lancet 2000; 356(9226).

36. Parm Ü, Metsvaht T, Sepp E, Ilmoja ML, Pisarev H, Pauskar M, Lutsar I. Mucosal surveillance cultures in predicting grame-negative late-onset sepsis in neoanatal intensive care units. J Hosp Infect 2011; 78(4).

37. Hulzebos CV, van Zoonen AGJF, Hulscher JBG, Schat TE, Kooi EMW, Koehorst M, et al. Fecal bile salts and the development of necrotizing enterocolitis in preterm infants. PLoS One 2017; 12(1); e0168633

38. Schat TE. Schurink M, van der Laan ME, Hulscher JBF, Hulzebos CV, Bos AF, et al. Near-infrared spectroscopy to predict the course of necrotizing enterocolitis. PLoS One 2016; 11(5): e0154710

39. Heida FH*, Schat TE*, Schurink M, van der Laan ME, Hulzebos CV, Bos AF, et al. The relation between splanchnic ischaemia and intestinal damage in necrotizing enterocolitis. Arch Dis Child Fetal Neonatal Ed 2016; 101(6).

40. Schurink M, Kooi EMW, Hulzebos CV, Kox RG, Groen H, Heineman E, et al. Intestinal fatty acid-binding protein as a diagnostic marker for complicated and uncomplicated necrotizing enterocolitis: a prospective cohort study. PLoS One 2015; 10(3): e0121336.

41. World Health Organization. Training course on child growth assessment: WHO child growth standards. 2008.

42. de Goffau MC, Luopajärvi K, Knip M, llonen J, Ruohtula T, Harkonen T, et al. Fecal microbiota composition differs between children with $\beta$-cell autoimmunity and those without. Diabetes 2013; 62(4).

43. Bartram AK, Lynch MD, Stearns JC, Moreno-Hagelsieb G, Neufeld JD. Generation of multimillionsequence 16S rRNA gene libraries from complex microbial communities by assembling paired-end illumine reads. Appl Environ Microbiol 2011; 77.

44. Bokulich NA, Joseph CM, Allen G, Benson AK, Mills DA. Next-generation sequencing reveals significant bacterial diversity of botrytized wine. PloS One 2012; 7; e36357.

45. Masella AP, Bartram AK, Truszkowski JM, brown DG, Neufeld JD. PANDAseq: paired-end assemble for illumine sequences. BMC Bioinformatics 2012; 13(31). 
46. Ludwig W, Strunk O, Westram R, Richter L, Meier H, Yadhukumar, Buchner A, et al. ARB: a software environment for sequence data. Nucleic Acids Res 2004; 32(4).

\section{Declarations}

Clinical trial informative: see attachment

Ethical approval: This study is part of a prospective observational trial (CALIFORNIA trial; registered as NTR4153 in the Dutch trial registry, and approved by the Medical Ethics Committee of the University Medical Center Groningen),

Financial disclosure: FHH and AGJFvZ were financially supported by a grant from the Junior Scientific Master Class of University of Groningen. FHH received a grant from the Gratama Foundation.

Conflict of Interest: No conflicts of interest have to be disclosed.

Address correspondence to: F.H. Heida, University of Groningen, University Medical Center of Groningen, Hanzeplein 1, 9713 GZ Groningen, [fardou@gmail.com], +31613935058.

Availability of data and materials:

The datasets during and/or analyzed during the current study are available on reasonable request.

\section{Author consent and contribution:}

F.H. Heida: Dr. Heida conceptualized and designed the microbiome study, performed data analysis, drafted the initial manuscript, and approved the final manuscript as submitted.

E.M.W. Kooi: Dr. Kooi, performed data analysis, reviewed and revised the manuscript and approved the final manuscript as submitted.

T.Y. Nguyen: BSc Nguyen performed the DNA extraction of the fecal samples, reviewed and revised the manuscript and approved the final manuscript as submitted.

J.B.F. Hulscher: Dr. Hulscher conceptualized and designed the study, supervised the study, reviewed and revised the manuscript and approved the final manuscript as submitted.

G.J.F. van Zoonen: Dr. van Zoonen designed and conceptualized the CALIFORNIA study, performed sample collection, reviewed the manuscript and approved the final manuscript as submitted.

A.F. Bos: Prof. dr. Bos conceptualized and designed the study, reviewed and revised the manuscript and approved the final manuscript as submitted.

H.J.M. Harmsen: Dr. Harmsen supervised the study (laboratory), reviewed and revised the manuscript and approved the final manuscript as submitted. 
M. C. de Goffau: Dr. de Goffau performed data analysis (including statistical analysis), supervised the microbiome study, reviewed and revised the manuscript and approved the final manuscript as submitted.

Acknowledgments: We would like to thank all the parents of subsequent neonates for participation in the study. Also, we would like to thank all the staff and students who were involved in the inclusion and sample collection of the study. We greatly acknowledge the help of C. Bus-Spoor and R.H.J. Tonk for technical assistance of the study.

\section{Tables}

Due to technical limitations, Table 1-2 are provided in the Supplementary Files section.

\section{Figures}
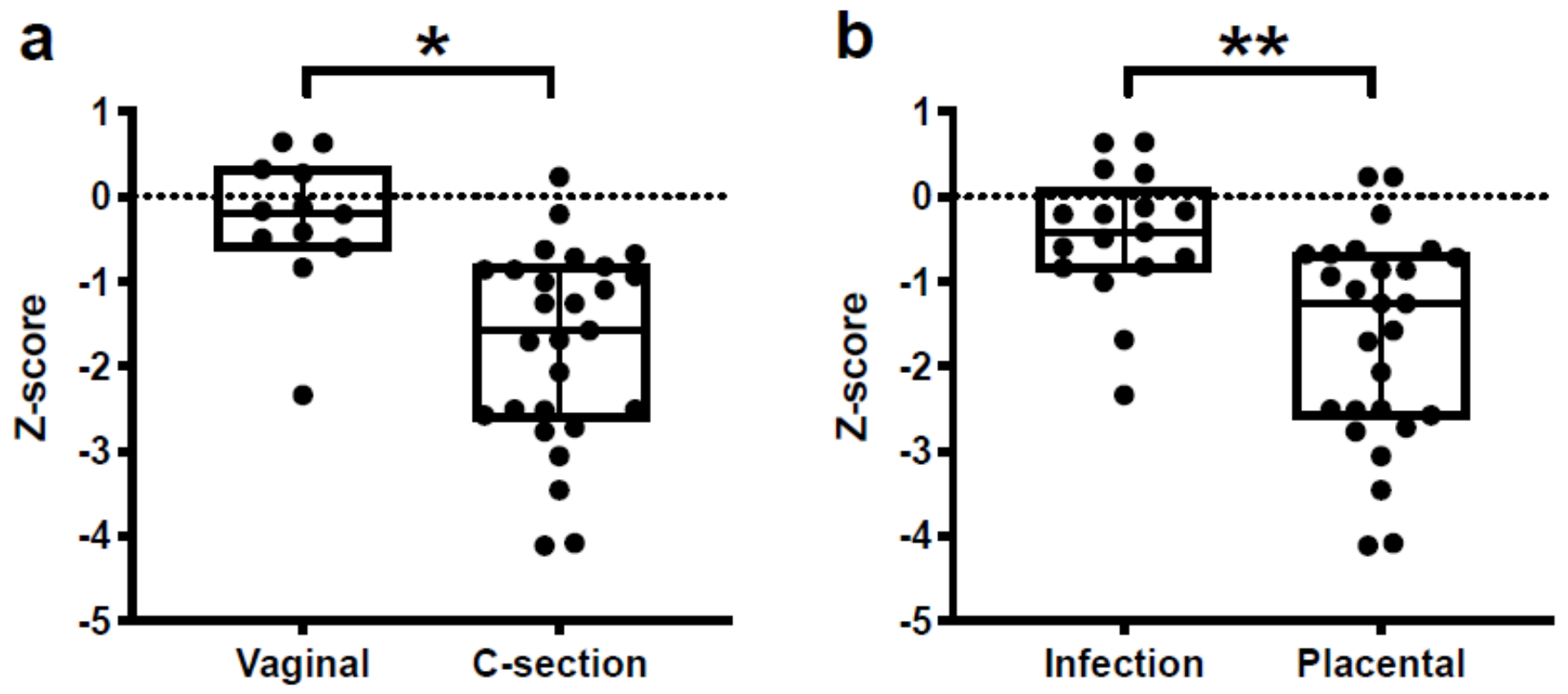

Figure 1

Boxplot of the relation between birthweight $z$-scores and the a) mode of delivery and b) the underlying cause of prematurity 


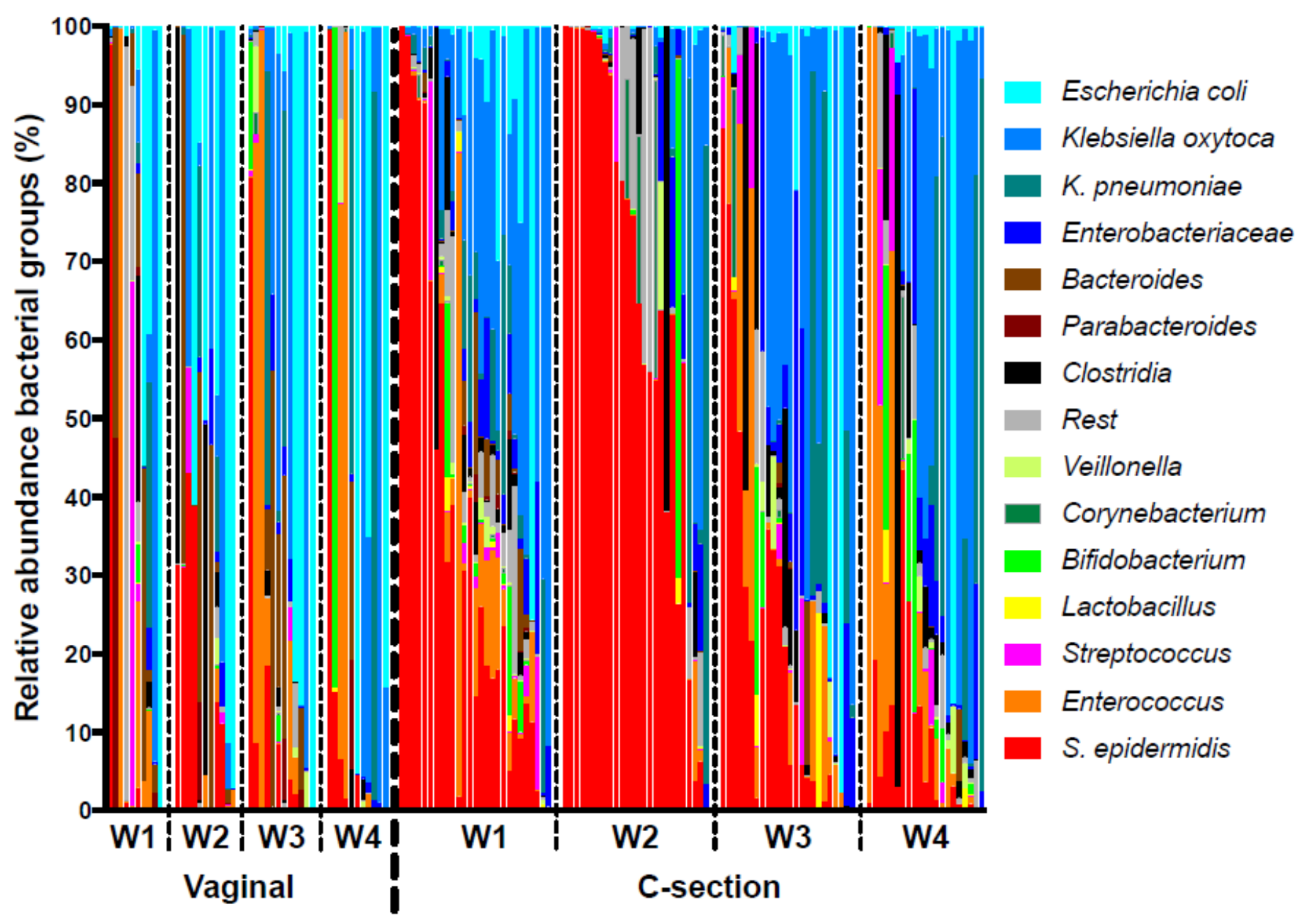

Figure 2

Overview relative abundance main bacterial groups per week (W1-4) in preterm infants delivered vaginally or by CS 

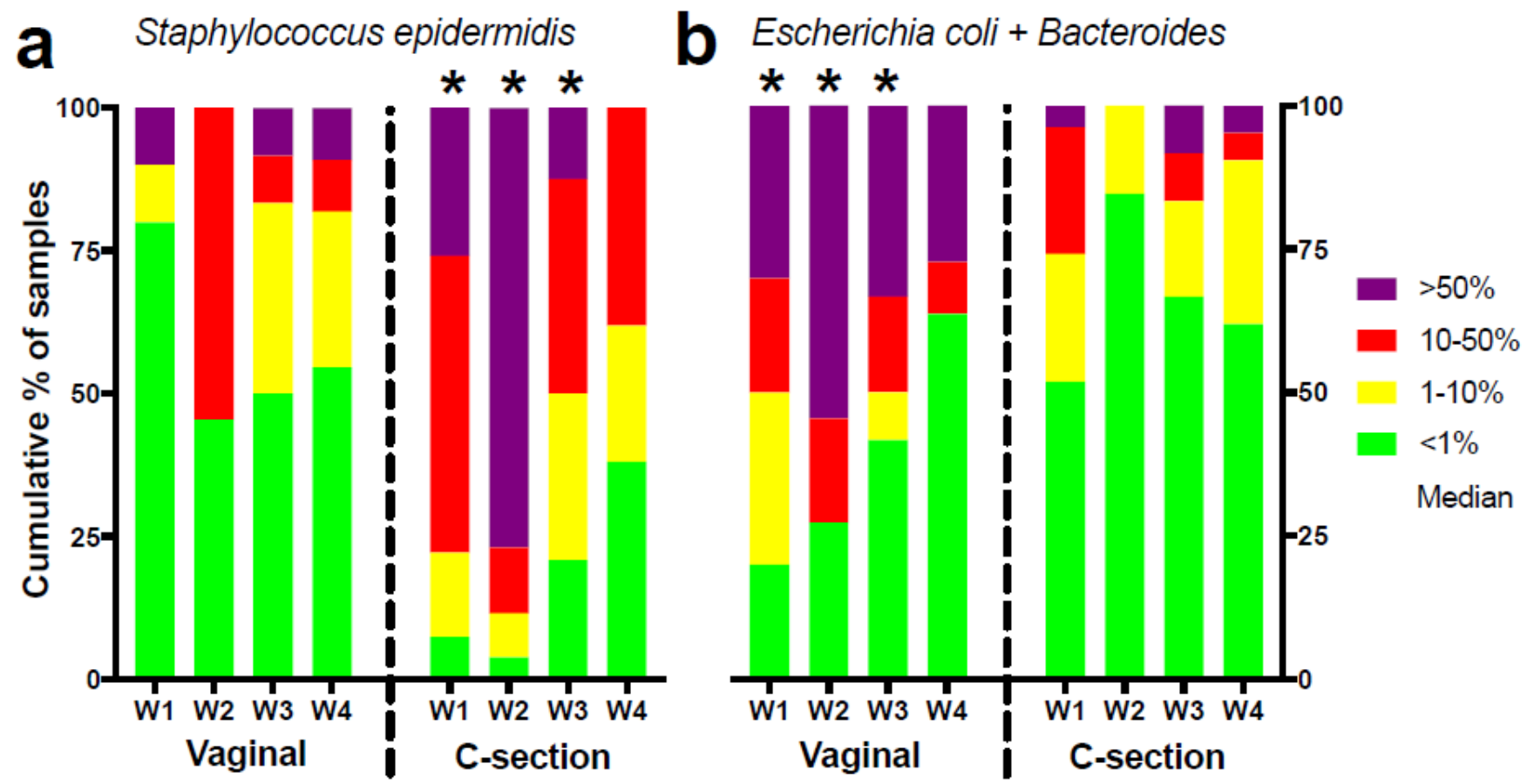

Figure 3

Prolonged effect of the mode of delivery on the faecal microbiota composition during the first weeks of life of preterm infants

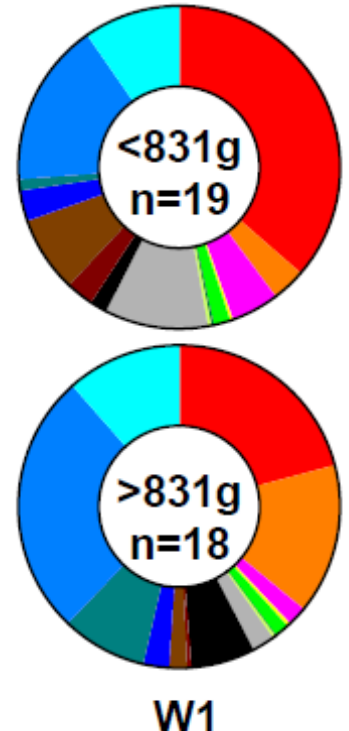

W1
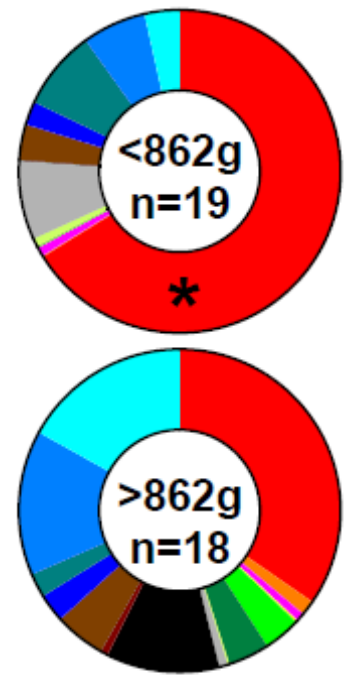

W2
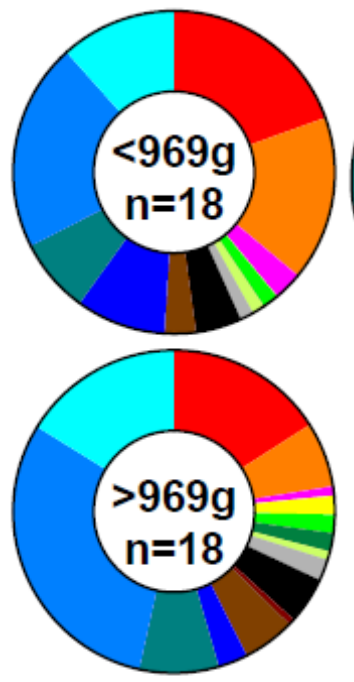

W3
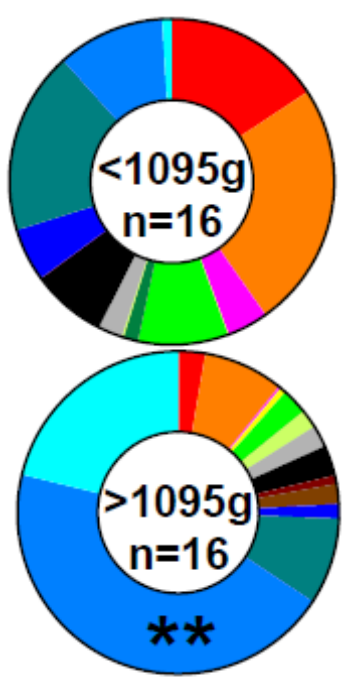

W4

Figure 4

Average abundance of bacterial groups in samples below or above the median weight of each week 


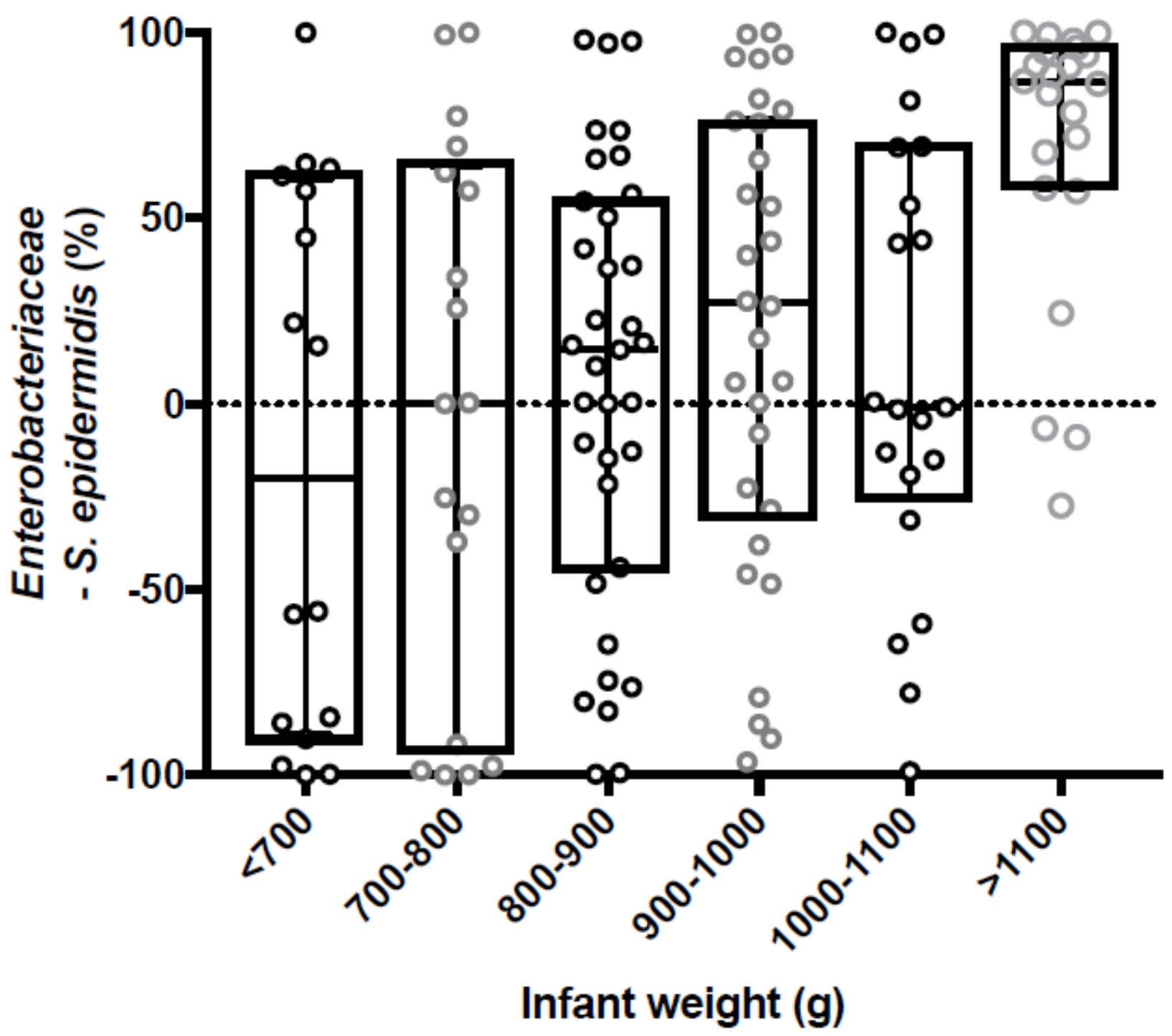

Figure 5

Boxplot of infant microbiota composition in relationship to infant weight

\section{Supplementary Files}

This is a list of supplementary files associated with this preprint. Click to download.

- Table1.pdf

- Table2.pdf

- SupplementalTable1.pdf

- SupplementalDatafile1.docx 